\title{
DEVELOPMENT AND TESTING OF A PROTOTYPE TUNER FOR THE CEBAF UPGRADE CRYOMODULE*
}

\author{
G. Davis ${ }^{\dagger}$, J. Delayen, M. Drury, E. Feldl, TJNAF, Newport News, VA 23606, USA
}

\begin{abstract}
An upgrade cryomodule is being developed for CEBAF at Jefferson Lab. The high-gradient, low-current operation of the superconducting cavities puts stringent requirements on the accuracy of the frequency tuner: a resolution of $1 \mathrm{~Hz}$ over a range of $400 \mathrm{kHz}$. We have developed and tested such a tuner; it consists of a steppermotor-driven coarse tuner, operating in series with a piezo-actuator-driven fine tuner. Tuner test procedures and results are summarized, including frequency resolution, range, and linearity (i.e. frequency vs. tuner position), as well as microphonic effects resulting from tuner operation.
\end{abstract}

\section{TUNER REQUIREMENTS AND SPECIFICATIONS}

The tuning system has been divided into two parts: a coarse tuner with a range of $+/-200 \mathrm{kHz}$ and resolution of $100 \mathrm{~Hz}$ that is expected to be used infrequently, and a fine tuner with a range of $+/-1 \mathrm{kHz}$ and resolution of $1 \mathrm{~Hz}$ that will be used during normal operation [1].

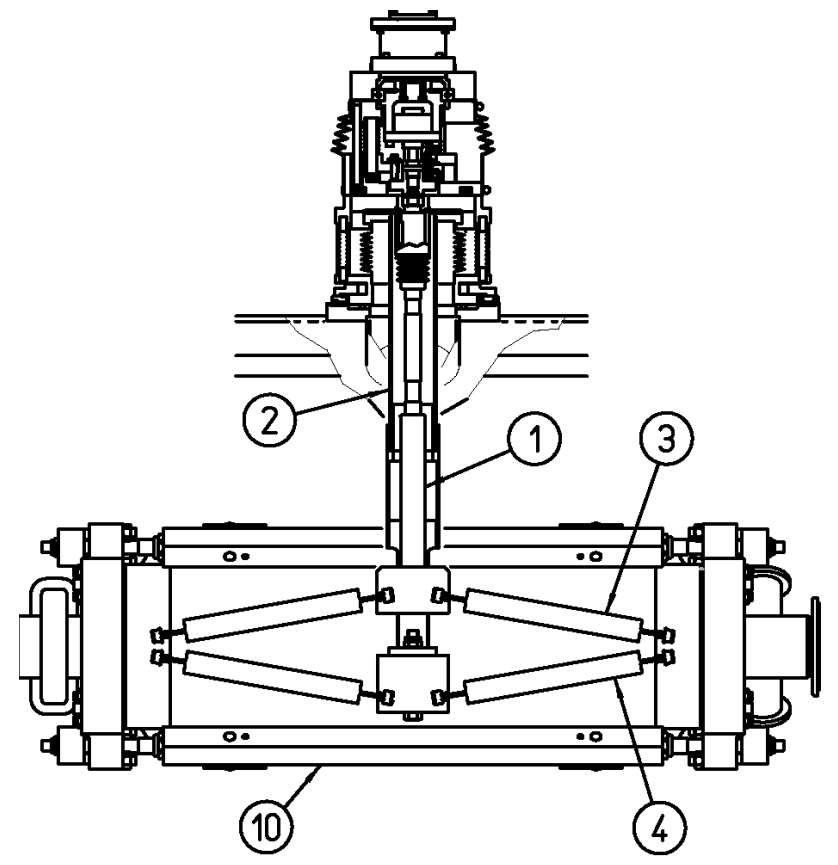

Fig. 1: CEBAF Upgrade Tuner

\footnotetext{
* This work was supported by the U.S. DOE Contract No DE-AC0584-ER40150.

delayen@jlab.org
}

\section{MECHANICAL DESIGN}

The fine tuner makes use of piezoelectric actuators for continuous small corrections to the cavity frequency. A stepping motor, via a harmonic drive reducer and ball screw, actuates the coarse tuner. In order to make them accessible for maintenance and repair, both systems are at ambient temperature, and are outside the vacuum enclosure. The tuning motion of the drive system enters the cryostat through two thin-wall concentric tubes (Items $1 \& 2$, Fig. 1). The tubes are connected to the upper and lower arms (Item $3 \& 4$, Fig. 1) of a scissors type jack. The axial motion of the tubes translates into a linear stroke parallel to the cavity center line [1]. To eliminate friction, motion within the vacuum is by flexing Ti plates.

\section{PROTOTYPE TESTING}

A prototype of the tuner, coupler, and cavity were tested at $2 \mathrm{~K}$ in a Horizontal Test Bed (HTB) [2] constructed for this purpose. The cavity was locked to its resonant frequency with a phase locked loop (PLL). Tuner range and hysteresis (cavity frequency vs. tuner position, Fig. 2) were measured with a frequency counter. Tuner resolution (Fig. 3) was measured using a Cavity Resonance Monitor (CRM) instrument: a custom-built IQ demodulator which outputs a voltage proportional to the difference in frequency between the PLL and a reference LO (local oscillator). Test results are summarized in Table 1. Additionally, tests were performed on both tuners to analyze possible tuner-induced microphonics; these frequency shifts measured less than $0.3 \mathrm{~Hz}$ peak. Finally, the dynamic response of the fine tuner was tested.

\subsection{Coarse Tuner}

The stepper motor was driven using a micro-stepping indexer which drives the stepper motor in 1/125 fractions of a whole motor step ( $\mu$ step). Simultaneously, the cavity frequency was measured with a frequency counter. The cavity was exercised over the operating range of the tuner. This range is bounded at one end by the requirement to operate the cavity in tension, and at the other end by tuner mechanical limits. Testing showed a range of $343.8 \mathrm{kHz}$. The coarse tuner range was less than the $400 \mathrm{kHz}$ design specification due to mechanical interference within the test setup. Bench measurements showed adequate range.

The PLL remained locked over a $110 \mathrm{kHz}$ portion of the coarse tuner range. Over this range, the tuner linearity was within two percent of the average slope, except the first value following a tuner direction reversal. The deviation of this first value is due to hysteresis. 
Table 1: Tuner Specifications and Test Results

\begin{tabular}{|c|c|c|c|c|c|}
\hline \multicolumn{3}{|c|}{ Measurement } & Specification & Test Result & Comment \\
\hline \multirow{6}{*}{ 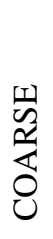 } & \multirow[t]{2}{*}{ Overall } & Range & $\pm 200 \mathrm{kHz}$ & $343.8 \mathrm{kHz}$ & Limited by test setup \\
\hline & & Resolution/Deadband & $100 \mathrm{~Hz}$ & $<2 \mathrm{~Hz}$ & S/N limited measurement \\
\hline & \multirow[t]{2}{*}{$50 \mathrm{kHz}$} & Hysteresis & Not Specified & $<3380 \mathrm{~Hz}$ & \\
\hline & & Repeatability & Not Specified & $<434 \mathrm{~Hz}$ & $198 \mathrm{~Hz}$ average \\
\hline & \multirow[t]{2}{*}{$700 \mathrm{~Hz}$} & Hysteresis & Not Specified & $<153 \mathrm{~Hz}$ & \\
\hline & & Repeatability & Not Specified & $<37 \mathrm{~Hz}$ & $17 \mathrm{~Hz}$ average \\
\hline \multirow{4}{*}{$\frac{1}{I}$} & \multirow[t]{2}{*}{ Overall } & Range & $\pm 1 \mathrm{kHz}$ & $2.4 \mathrm{kHz}$ & \\
\hline & & Resolution/Deadband & $1 \mathrm{~Hz}$ & $1 \mathrm{~Hz}$ & S/N limited measurement \\
\hline & \multirow[t]{2}{*}{$2.5 \mathrm{kHz}$} & Hysteresis & Not Specified & $933 \mathrm{~Hz}$ & \\
\hline & & Repeatability & Not Specified & $<329 \mathrm{~Hz}$ & $213 \mathrm{~Hz}$ average \\
\hline
\end{tabular}

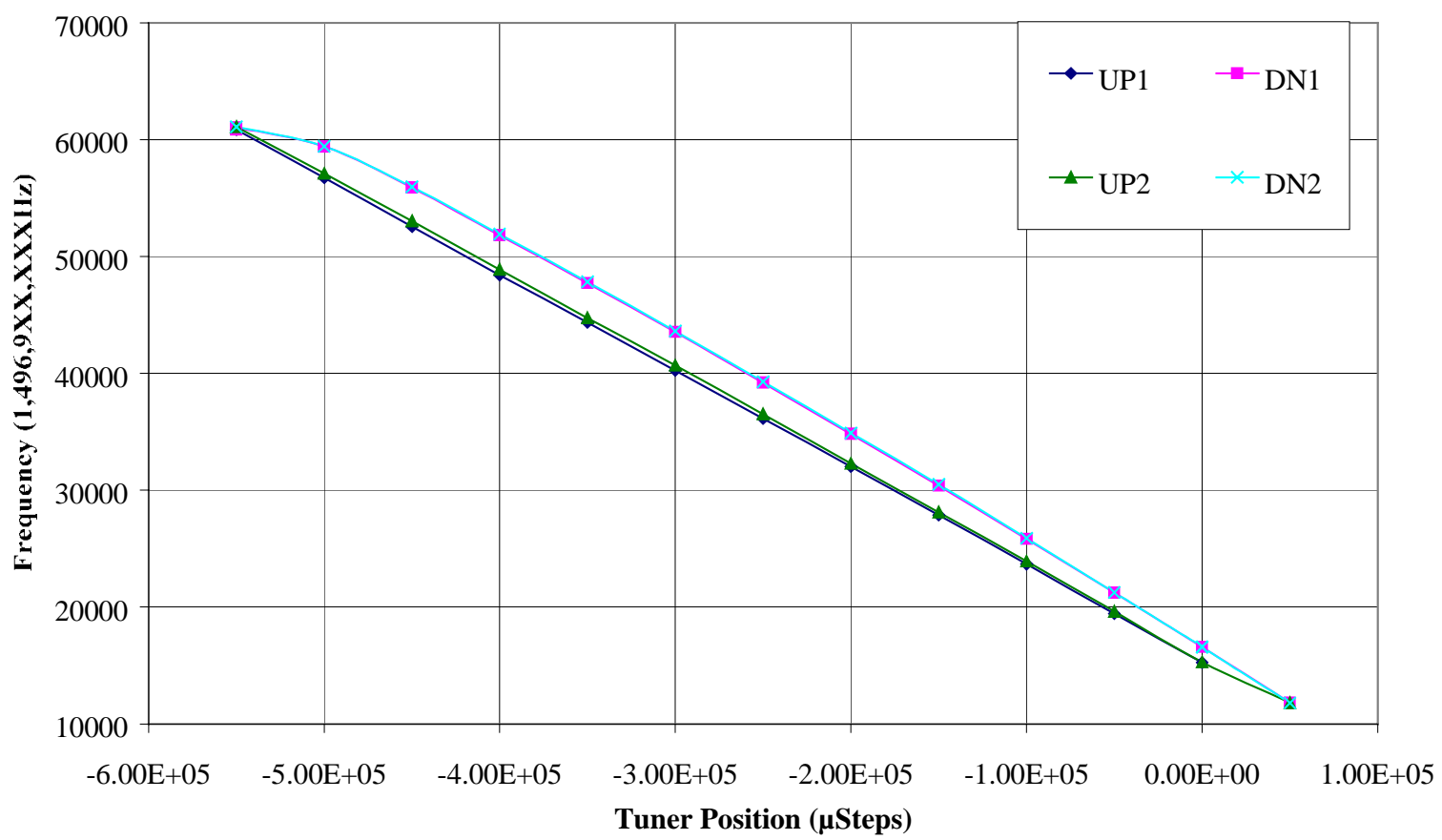

Figure 2: Coarse Tuner 50kHz Range Hysteresis Test

Two sets of hysteresis tests were performed: using a $50 \mathrm{kHz}$ range, then a $700 \mathrm{~Hz}$ range. Each test consisted of two complete cycles of the tuner position over the applicable range. The results of the $50 \mathrm{kHz}$ test are shown in Figure 2.

To test the tuner resolution and deadband, LabView software was used to drive the stepper motor back and forth by some integral number of motor steps. The software recorded the drive signal and the output of the CRM. Low pass filtering (single pole, $1 \mathrm{~Hz}$ ) was used to reduce high-frequency noise induced by cryogenic system pressure transients. Data was taken at a drive amplitude of two steps every 5 seconds $(0.1 \mathrm{~Hz})$, see Figure 3 . The amplitude of the response is approximately $2 \mathrm{~Hz} \mathrm{pp}$. Cryogenic system pressure fluctuations produced the noise and drift superimposed on the response square wave; lower response amplitudes were not recorded because of unusable $\mathrm{S} / \mathrm{N}$ ratio. Consistent response was observed, though not recorded, at an amplitude of $1 \mathrm{~Hz} \mathrm{pp}$.

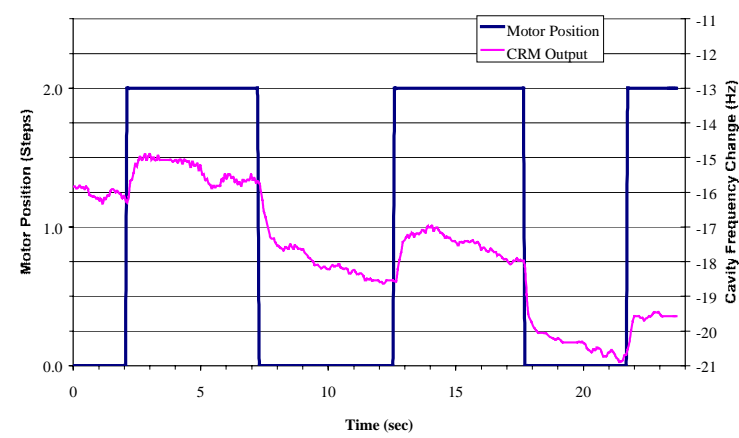

Figure 3: Coarse Tuner Resolution/Deadband Test 


\subsection{Fine Tuner}

The piezo actuators were driven using a DC voltage amplifier. Otherwise, tests of fine-tuner range, $2.5 \mathrm{kHz}$ hysteresis, and resolution/deadband were performed as described for the coarse tuner. All specifications for the fine tuner were met or exceeded, as summarized in Table 1.

The frequency response of the fine tuner is of interest for potential dynamic applications such as active vibration cancellation and, during pulsed operation, Lorentz detuning compensation. The magnitude and phase response of the fine tuner system was measured using a lock-in amplifier. A function generator was used to generate a sinusoidal signal into the power amplifier, driving the piezo actuator. The CRM was used to monitor the resulting sinusoidal modulation of the cavity resonance, which was locked to the PLL using low-power RF. The frequency of the tuner input was swept while the drive amplitude was maintained at $30 \mathrm{Vpp}$ (amplifier limited) across the piezo actuators. Concurrently, the lock-in amp measured the resulting amplitude and phase with respect to the function generator output. The results, shown in Figure 4, represent the frequency response of the entire system: tuner, cavity, and PLL.

There are several significant characteristics of the system response. The input variable is drive frequency, the output variable is cavity resonant frequency. Within the frequency range of interest $(<200 \mathrm{~Hz})$, the response varies over two orders of magnitude. Since the piezo can be driven to $150 \mathrm{Vpp}$, the system is capable of five times more output magnitude than this test produced. In order to accomplish this, alternate amplification schemes will be required. There are several points where the system response is so small that active vibration cancellation might not be feasible. Note, in particular, the local minimum at $141 \mathrm{~Hz}$, where the cavity resonant frequency could only be driven to deviate by $.6 \mathrm{~Hz}(\mathrm{rms})$. Finally, there is a resonance at $33.3 \mathrm{~Hz}$ (this resonance was previously identified in microphonics vibration testing[3]). The fine tuner response was swept to $1000 \mathrm{~Hz}$, suggesting that the transient response of the tuner to pulsed-type input drive signals should be very good.

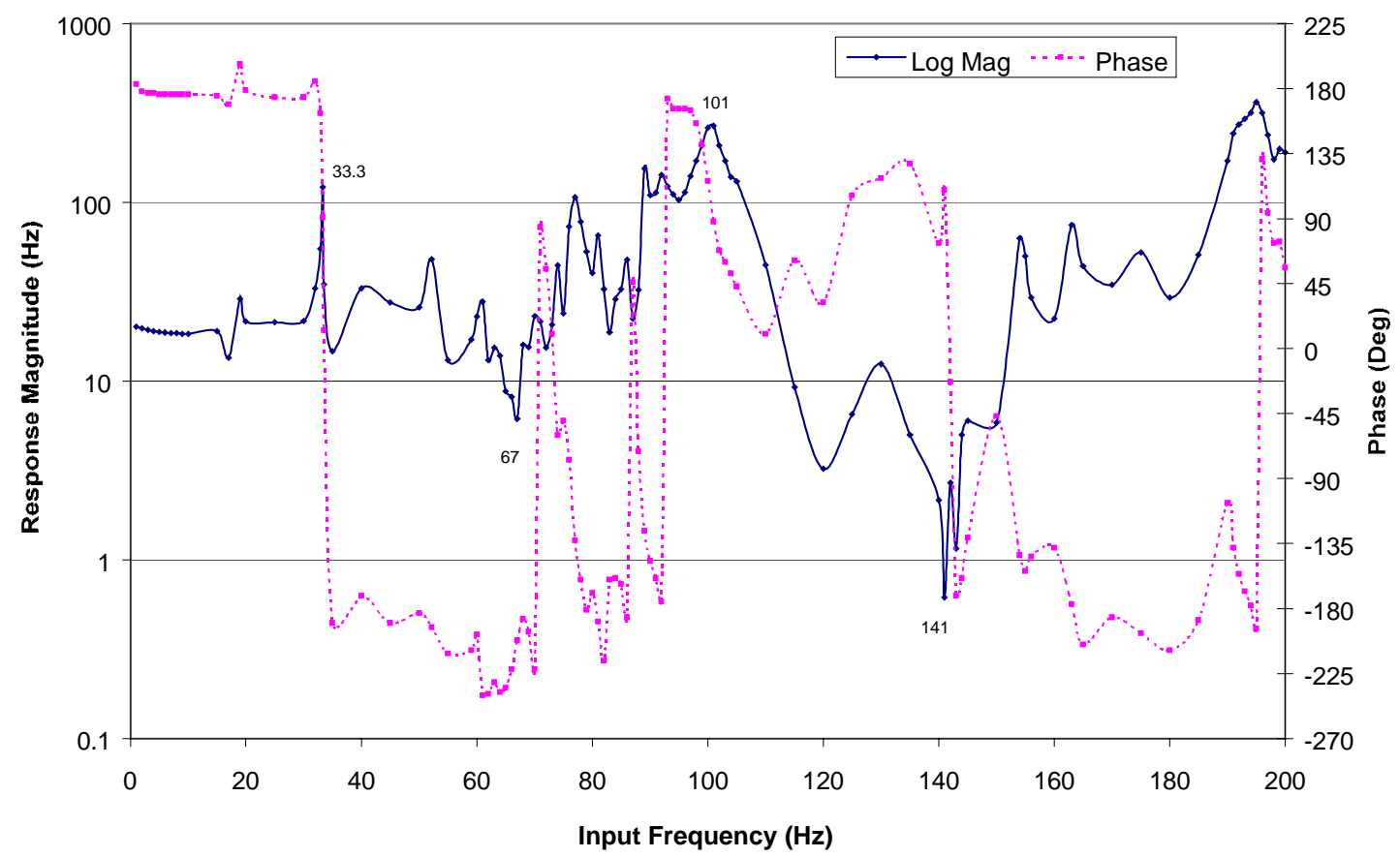

Figure 4: Fine Tuner Bode Plot

\section{CONCLUSIONS}

The tuner met or exceeded all design specifications. The design has been refined to meet cost-down goals. A new prototype has been constructed and is now being tested.

\section{REFERENCES}

[1] J. Delayen, L. Doolittle, E. Feldl, V. Nguyen, "Frequency Tuning of the CEBAF Upgrade Cavities," PAC'99, New York, NY, April 1999.

[2] I. Campisi, et al, "CEBAF Upgrade Cryomodule Component Testing in the Horizontal Test Bed (HTB)," these proceedings.

[3] G. Davis, J. Delayen, M. Drury, T. Hiatt, C. Hovater, T. Powers, J. Preble, "Microphonics Testing of the CEBAF Upgrade 7-Cell Cavity," these proceedings. 